\title{
Reduced Complexity Diversity Combining and Adaptive Equalization Using Interpolated Channel Estimates with Applications to Cellular Mobile Radio Channels.*
}

\author{
Tugay Eyceöz and Alexandra Duel-Hallen \\ Dept. of Electrical \& Computer Engineering, \\ North Carolina State University, Box 7911, \\ Raleigh, NC 27695-7911 \\ E-mail: teyceoz@eos.ncsu.edu, sasha@eos.ncsu.edu
}

\begin{abstract}
In this paper, we investigate an antenna diversity combiner-equalizer receiver structure to combat multipath fading in Cellular Mobile Radio (CMR) communications. Our system specifications are compatible with the U.S. digital cellular system. The technique utilizes block adaptation and linear or decision feedback equalization. The receiver offers complexity reduction relative to previously proposed block adaptation methods [1,2] without sacrificing performance.
\end{abstract}

\section{Introduction}

The main objective in the design of digital communication systems is to achieve the highest possible transmission rate with acceptable level of reliability. In CMR communications the basic problem is the time dispersion introduced by the Rayleigh fading multipath channel. Thus, the signal dispersion caused by frequency selective multipath fading and the time-varying signal propagation resulting from vehicular motion introduce intersymbol interference (ISI) and random doppler fading $[3,4,5,6]$. In order to combat intersymbol interference (ISI) and rapid time variations in CMR communications occurring over frequency selective fading multipath channels, we investigated a diversity combiner-adaptive equalizer receiver structure. Specifically, we used diversity reception, known to combat fast fading, along with adaptive equalization, known for mitigating the effects of ISI, for our receiver structure. As a diversity reception technique we used space diversity with multiple antennas, spaced sufficiently far apart so that their received signals fade independently.

It is important to consider channel fading in the design of an adaptive equalizer. In CMR communications where the channel is fast fading, combiner equalizer parameters can be calculated periodically based on channel estimates or updated using an adaptive equalization algorithm. We used the former approach. Specifically, block adaptation using known training sequences is used for our CMR communications system. In contrast to continuous adaptation, a block adaptation algorithm computes and changes the receiver parameters at the beginning of each data frame. The start of each data frame contains a known training sequence which is used to estimate the channel impulse response (CIR). However, for a fast fading channel, the CIR estimate obtained from training at the beginning of a data frame should be updated several times during the data frame. This time-varying CIR is determined by interpolating a set of estimated CIR

\footnotetext{
*This research was supported by NSF grant NCR-9410227.
}

values. Then the combiner-equalizer parameters are adaptively computed using interpolated channel estimates from each of the $L$ diversity channels, yielding the optimum performance in the MMSE sense.

In [2], Lo et al. used the same block-adaptive strategy. However, their receiver structure was different. They used an individual fractionally spaced feedforward filter for each diversity path and one common symbol spaced feedback filter. On the other hand, in [7], Balaban and Salz derived a symbol spaced Minimum Mean Square Error (MMSE) optimum receiver structure. They also combined diversity reception with adaptive equalization into a single receiver. Implementing this receiver requires knowledge of actual channel characteristics, since this receiver features a set of matched filters for each diversity path. The additional parts of their optimum receiver structure consist of tapped-delay line filters used for processing the sum of the output samples from the matched filters prior to detection. We combined blockadaptive strategy approach with the receiver structure similar to that of Balaban and Salz. Our receiver has fractionally spaced matched filter for each diversity branch followed by a single symbol spaced Linear Equalizer (LE) or Decision Feedback Equalizer (DFE). This receiver structure has lower computational complexity than that of Lo et al., and does not require prior knowledge of channel parameters as that of Balaban and Salz.

In the following sections we describe our CMR communication model and discuss simulation results.

\section{CMR System Description}

A block diagram of the CMR communications system is presented in Figure 1. Our system specifications are compatible with the U.S. digital cellular system. A narrowband time division multiple access (TDMA) channel access method accommodates three users in a band of $30 \mathrm{kHz}$ in each direction, with $8 \mathrm{kbits} / \mathrm{s}$ user. The $\pi / 4$-shifted DQPSK modulation method is used. The transmit and receive filters have square root raised cosine frequency response characteristics with a $35 \%$ rolloff factor. In this paper a discrete-time representation is used which corresponds to sampling all signals at $T / 2$, where $T$ is a symbol interval.

At the $l^{\text {th }}$ diversity branch, the received signal is

$$
\begin{aligned}
V_{l}(t) & =S(t) * h_{l}(t)+N_{l}(t) \\
& =\sum_{k} S(k T) h_{l}(t-k T)+N_{l}(t)
\end{aligned}
$$




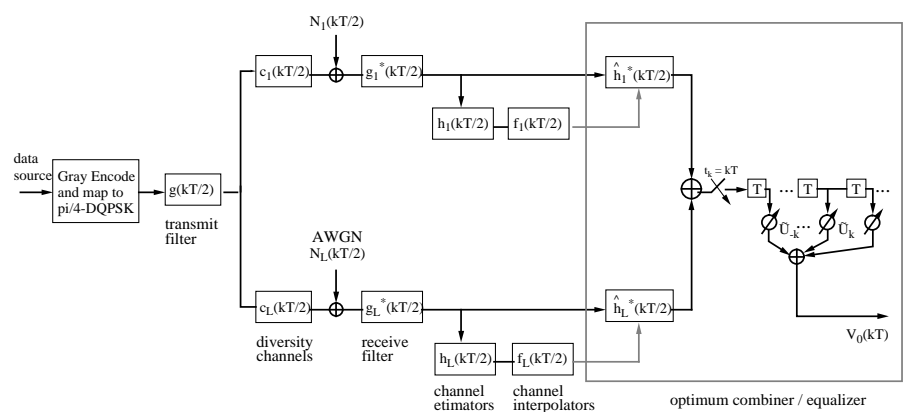

Figure 1: CMR communications system with linear block adaptive equalization

where $S(k T)$ is the $\pi / 4$-shifted DQPSK data sequence at time $k T, N_{l}(t)$ is the AWGN with the variance of $\sigma_{n}^{2}$, and $h_{l}(t)$ is the channel impulse response (CIR) which also includes the transmit and received filter responses.

\section{Channel Model}

For each diversity branch, the CMR channel is modeled in complex baseband representation as an FIR filter in the form of a $\mathrm{T} / 2$-spaced tapped-delay-line (TDL) as shown in Figure 2. The TDL consists of three complex tap coefficients, $c_{l}^{i}(k T) \mathrm{i}=1,2,3$, each representing a distinct multipath. The multipath power delay profile (MPDP) of the channel is specified with a three-paths "mountainous terrain" model with relative rms powers of $0,-5$, and $-15 \mathrm{~dB}$. Each channel tap coefficient having Rayleigh fading characteristics is simulated with the "modified Jakes model" [3, 8] using sixteen sinusoids with distinct Doppler frequencies up to a maximum Doppler frequency, fdm of $100 \mathrm{~Hz}$. Furthermore, complex additive white gaussian noise (AWGN) with variance $\sigma_{n}^{2}$ is added to the transmitted signal at the output of each diversity channel. These noise components are independent.

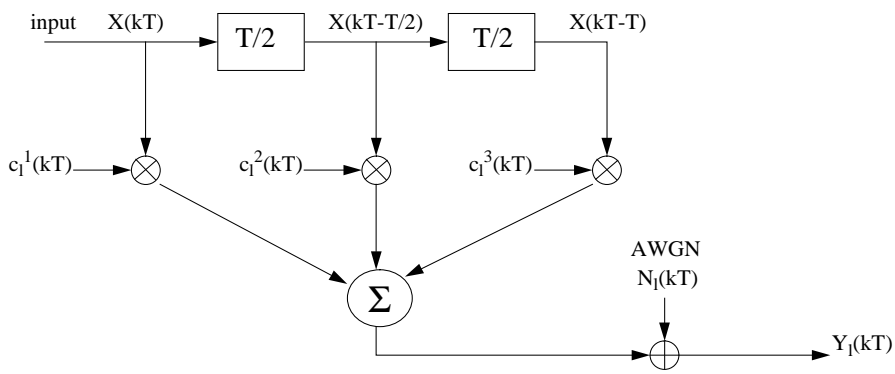

Figure 2: T/2-spaced TDL with three-paths "mountainous terrain" channel model at $l^{\text {th }}$ diversity branch

\section{Signaling Format}

The signaling format of alternating training and data sequences within a long TDMA time slot is used. Each data frame consists of a training sequence of length $N_{t}$ symbols followed by an uncorrelated data sequence of length $N_{d}$ symbols. Therefore, the number of symbols in each transmitted frame is

$$
\eta=N_{d}+N_{t}
$$

Since CIR estimates are updated at the beginning of each data frame, this type of periodic training improves the adaptation problem. However, it introduces some system overhead, which is defined as the percentage data throughput:

$$
\% T=\frac{100 N_{d}}{N_{d}+N_{t}} \%=\frac{100 N_{d}}{\eta} \%
$$

This signaling strategy is simpler than proposed narrowband TDMA signaling format. For example, some signaling information such as synchronization, guard and ramp times, and other control signals are not included in our time slot. Therefore, we ignore this overhead in our analysis.

\section{Block Adaptation-Channel Estimation and Interpolation}

In this section, we describe the channel estimation procedure which is performed for each diversity branch. We omit the branch index to simplify notation. To estimate the channel impulse response (CIR), block adaptation using a known training sequence is employed [2]. This technique is optimum under the assumption that the CIR is fixed during the training period. In our system, the overall CIR length, $N_{c}$, is fixed to six symbol periods of which five symbol periods are due to transmit and receive filter impulse responses, and one symbol period is due to the actual CIR of the CMR channel. The training sequence is 15 symbols long as suggested in [2].

During a training sequence, the overall CIR estimate, $\boldsymbol{h}$, is obtained from the Block Least Squares (BLS) solution described in [2]. The channel estimate is timevarying and changes during the data frame. After training, block-adaptive strategy computes the time-varying CIR by interpolating a set of estimated CIR values [2]. The relative positions of the estimated and interpolated CIR samples within the TDMA time slot are illustrated in Fig 3.

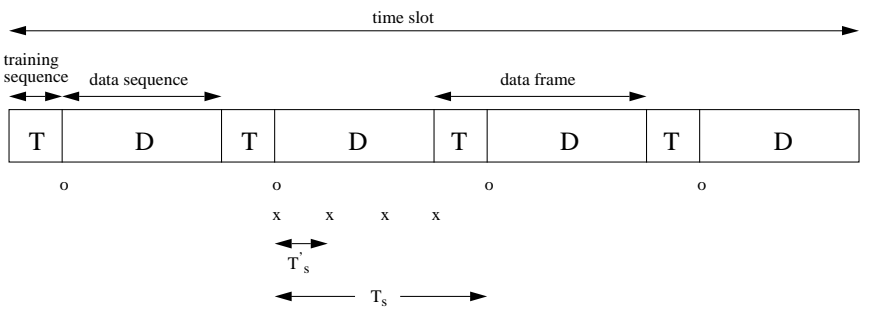

Figure 3: CIR interpolation within a TDMA time slot. 'o'estimated CIR sample at the end of a training sequence, $\mathrm{Q}=4$, 'x'-interpolated CIR in the middle data frame, $R=$ $T_{s} / T_{s}^{\prime}=4$

To satisfy Nyquist's sampling criterion, the sampling rate of the CIR estimates is required to be

$$
f_{s} \geq 2 f_{d m}
$$

where $f_{d m}$ is the maximum Doppler frequency. This indicates that CIR estimates have to be calculated at least as often as $2 f_{d m}$. Furthermore, the normalized sampling rate of the CIR estimates is defined as

$$
\bar{f}=\frac{f_{s}}{2 f_{d m}}=\frac{1}{2 f_{d m} \eta T} \geq 1
$$


i.e., for the maximum Doppler frequency of $f_{d m}=100 \mathrm{~Hz}$ and symbol rate of $\frac{1}{T}=24 \mathrm{ksps}$, the required frame length should be $\eta=N_{t}+N_{d} \leq 120$ symbols.

This block adaptation strategy was previously published [2], but its utilization in the receiver structure is new as described below.

\section{Receiver Structures}

We used interpolated CIR estimates, $\hat{\boldsymbol{h}}_{\boldsymbol{l}}$, from each of the $\mathrm{L}$ diversity channels to compute the receiver parameters yielding the optimum performance in the MMSE sense. The optimal receivers are derived under the assumption that the channel response $h_{l}(t)$ can be estimated perfectly, i.e., $\hat{h}_{l}(t)$ is given by the actual response $h_{l}(t)$.

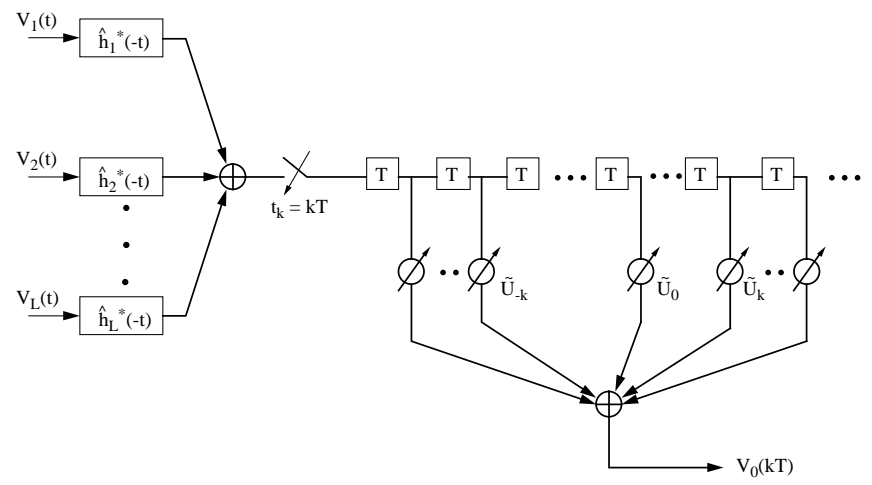

Figure 4: Optimum diversity combiner linear equalizer structure

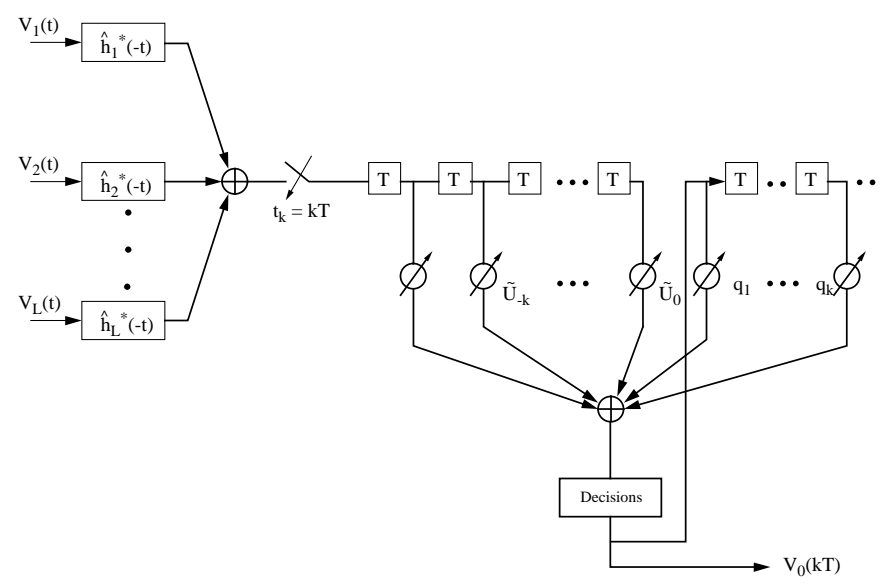

Figure 5: Optimum diversity combiner decision feedback equalizer structure

It was shown in [7] that first step in the implementation of the optimum linear or decision feedback equalizer is to pass the received signal $V(t)$ through a matched filter associated with the $l^{\text {th }}$ diversity channel. The outputs of the matched filters are then summed and processed using a symbol-spaced decision structure. The optimal configurations are depicted in Figures 4 and 5 for the linear and the decision feedback

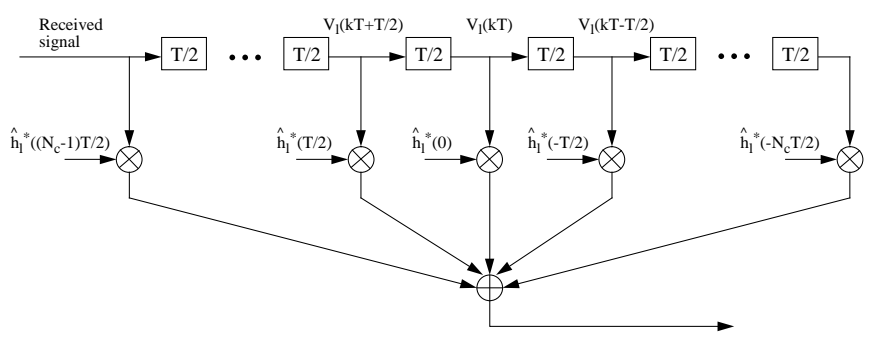

Figure 6: Fractionally spaced Matched Filter for the $l^{\text {th }}$ diversity branch

for equalizers, respectively. Moreover, the tap coefficients of these equalizers are derived as in [7]. In [7], known channel responses are assumed. However, in the adaptive implementation, channel responses need to be estimated to determine responses of the matched filters for each branch. We implemented these matched filters as fractionally spaced (specifically $T / 2$ spaced), transversal filters as seen in Figure 6 . The filter tap coefficients are determined by the current channel estimates obtained as described in the previous subsection. The overall length of the matched filter is six symbol periods which is the length of the CIR estimate.

The computational complexity reduction of the new structure results from employing a single equalizer following a diversity combiner. Previously proposed adaptive receivers featured separate fractionally spaced equalizers associated with each diversity branch. Calculation of the equalizer coefficients is significantly simplified. The computational complexity is independent of the number of antenna elements as opposed to linear dependence in previous implementations $[1,2]$.

\section{Simulation Results}

The CMR communication system is modeled with equivalent complex baseband signals and simulated through extensive use of Monte Carlo approach. Optimum diversity combiner linear equalizer with $N=5$ taps is denoted as ODCLE( 5,0$)$, and optimum diversity combiner decision feedback equalizer with $N_{f}=3$ taps in the forward filter and $N_{b}=2$ taps in the feedback filter is denoted as $\operatorname{ODCDFE}(3,2)$.

Numerical results illustrate several performance characteristics of the proposed receivers. System performance improves with more frequent CIR training while reducing the system throughput. For ODCLE $(5,0)$ the average BER performance as a function of the normalized sampling rate, $\bar{f}$, for different values of the maximum Doppler frequency is illustrated in Figure 7 for the case of $S N R=20 \mathrm{~dB}$ and $L=1$. We see from this figure that the average BER monotonically decreases for increasing values of $\bar{f}$ for high Doppler frequencies. However, when DFE is used instead of LE, we observe the decreasing BER for all Doppler frequencies as seen in Figure 7 for ODCDFE(3,2). DFE is superior to LE for all Doppler shifts since DFE is more appropriate for channels with spectral nulls and it does not suffer from noise enhancement.

The average BER performance as a function of the nor- 


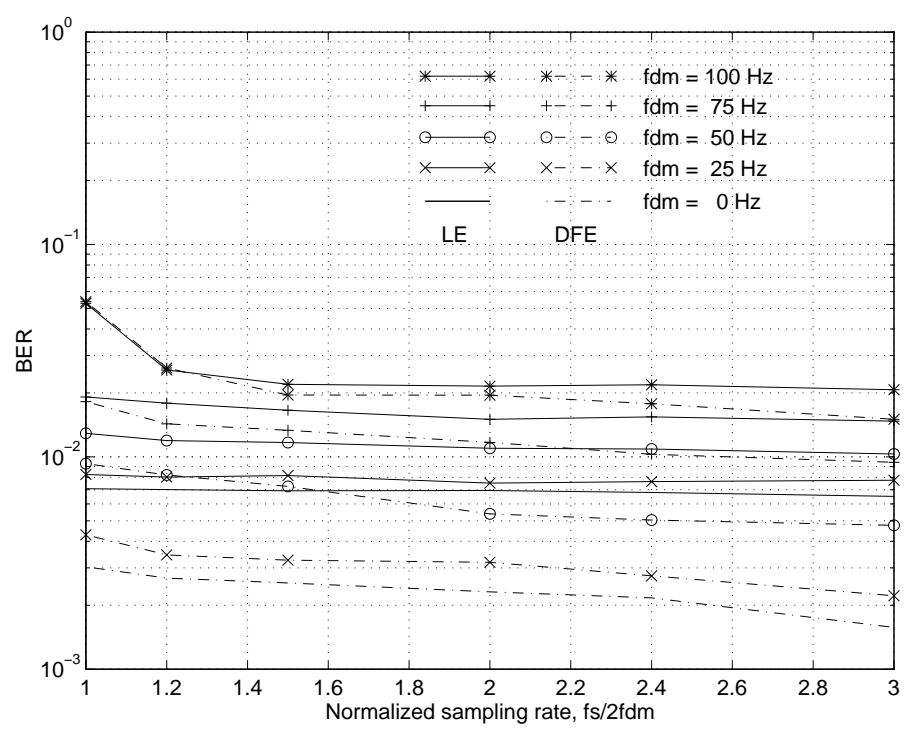

Figure 7: Average BER as a function of normalized sampling rate, $\bar{f}$, and the maximum Doppler frequency, $f_{d m}$ for ODCLE $(5,0)$ and ODCDFE $(3,2)$

malized sampling rate, $\bar{f}$, for the number of diversity channels, $L$, is illustrated in Figure 8 for the case of $S N R=20$ $\mathrm{dB}$ and the maximum Doppler frequency, $f_{d m}=100 \mathrm{~Hz}$. It can be seen from the figure that the system performance improves with increasing $\bar{f}$ and $L$. However, for all diversity cases, the system performance at the Nyquist sampling rate (i.e., $\bar{f}=1$ ) is low. This poor performance is caused by insufficiently frequent training and by the severe aliasing distortion due to the interpolation filter which leads to poor tracking of the fast fading channel at each diversity branch. The system performance gains tend to flatten out at high sampling rates. The average BER improvement in Figures 7 and 8 is due to more frequent training as we increased the normalized sampling rate, $\bar{f}$. The performance improvement with increasing $\bar{f}$ results in an overhead of reduced system throughput, $\% T$ (see Eq.3). Specifically, at $\bar{f}=1.0$, the system throughput, $\% T$, is $87.5 \%$ and the processing delay, $D_{t}$, is $15 \mathrm{~ms}$ while at $\bar{f}=3.0, \% T$ is $62.5 \%$ and $D_{t}$ is 5 ms. Therefore, one can select the suitable normalized sampling rate by considering the trade-off between the system performance and the throughput/delay requirements. Our results for various Doppler frequencies and the number of diversity channels show that a value of $\bar{f}=2.0$ (i.e., twice the Nyquist sampling rate), with an associated $\% T=75 \%$ and $D_{t}=7.5 \mathrm{~ms}$, is a reasonable design choice for our CMR communications system.

Next, we discuss the system performance as a function of the average channel SNR. The normalized sampling rate is chosen to be $\bar{f}=2.0$ for the rest of the simulations. The average BER performance as a function of the average channel SNR for the number of diversity channels, $L$, and maximum Doppler frequency, $f_{d m}$, is illustrated in Figure 9 for ODCLE $(5,0)$ and ODCDFE(3,2). The high channel noise is the dominant reason for the poor system performance at low SNR values. Although the system performance improves

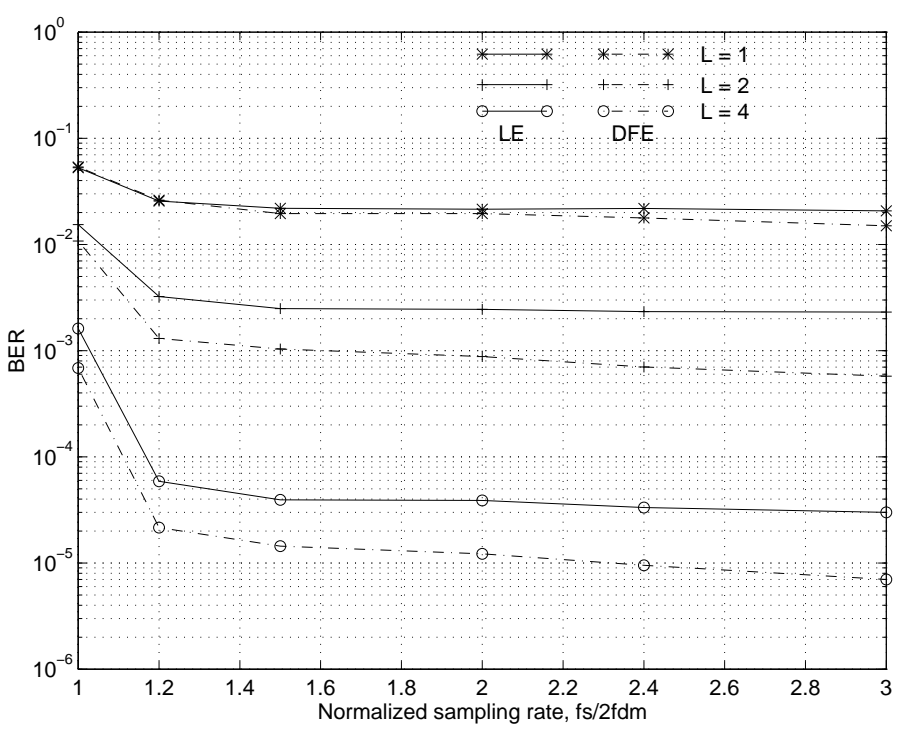

Figure 8: Average BER as a function of normalized sampling rate, $\bar{f}$, and diversity for both ODCLE(5,0) and OD$\operatorname{CDFE}(3,2)$ with $S N R=20 \mathrm{~dB}$ and $f_{d m}=100 \mathrm{~Hz}$

with increasing SNR, an irreducible BER was found to occur at high values of $\mathrm{SNR}>20 \mathrm{~dB}$. This is due to receiver limitations in estimation and tracking the rapid time variations in the fading channel. The use of diversity channels improves the system performance. Although it does not eliminate the error floor, it does reduce the level of the irreducible BER.

Although infinite number of equalizer taps is needed to cancel all ISI, we have to consider computational complexity involved in the calculation of the equalizer coefficients. Therefore, we searched for optimum equalizer length for our CMR communications system [1]. Our results indicate that the linear equalizer size of 5 symbol periods is optimum in terms of cancelling ISI for $L=1$. In addition, for diversity channels, $L=2$ and $L=4$, there is only less than $2 \mathrm{~dB}$ difference between the equalizer size of 5 and the infinite tap equalizer. Therefore, considering the computational complexity involved in calculation of the equalizer coefficients, the equalizer size of 5 symbol periods is a very good choice for our optimum diversity combiner and equalizer structure. Moreover, the equalizer length of 1 means only diversity is employed. We also note that when equalizer length is 1 , the system performance is severely degraded [1]. Hence, it is necessary to use an equalizer in addition to diversity combining to achieve good system performance.

In [2], Lo et al. used a different technique to calculate the receiver coefficients. Instead of using a matched filter for each diversity path, they used an individual fractionally spaced feedforward filter for each diversity path and one common symbol spaced feedback filter. The authors of [2] solved matrix equations with the order of $\left(L * N_{f}+N_{b}\right) \times\left(L * N_{f}+N_{b}\right)$ to calculate their DFE equalizer coefficients, where $N_{f}$ and $N_{b}$ are the number of forward and feedback taps respectively. Thus, the dimensions of the matrix depends on $L$. However, in our receiver structure, we solve matrix equations with the order of $N \times N$ for the LE and $\left(N_{f}+N_{b}\right) \times\left(N_{f}+N_{b}\right)$ for 


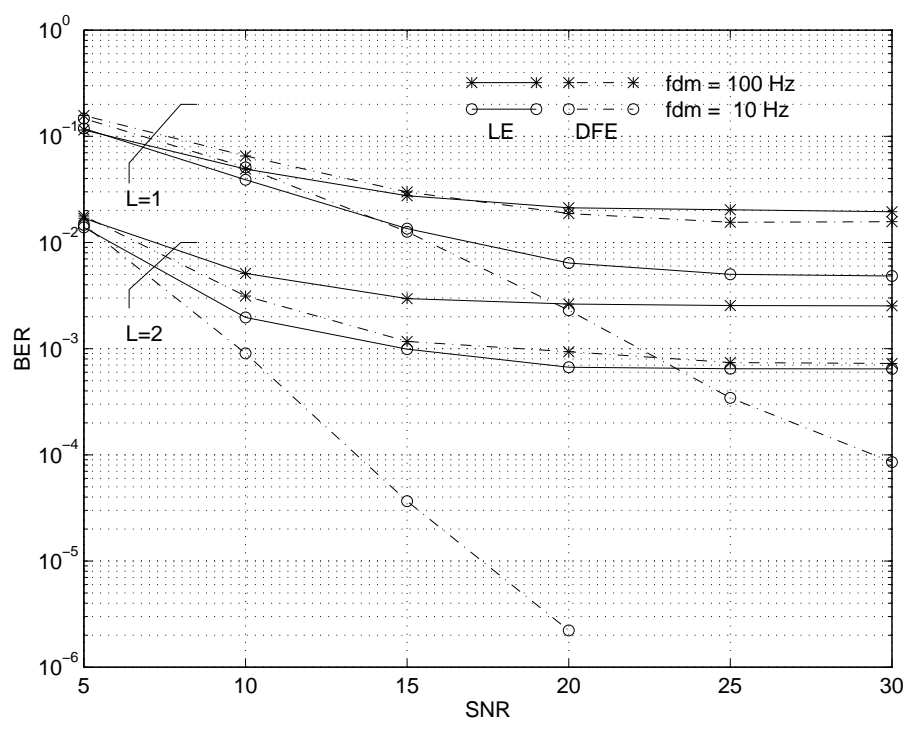

Figure 9: Average BER as a function of channel SNR, diversity and Doppler fading for ODCLE $(5,0)$ and ODCDFE $(3,2)$

the DFE. For example, Lo et al. chose $N_{f}=3$ and $N_{b}=2$. For the diversity order of $L=4$, they solved a $14 \times 14$ matrix equation. However, with the same number of taps (this number is also optimum for our system), we solve a $5 \times 5$ matrix equations regardless of diversity. Hence, computational complexity involved in calculation of the optimum diversity combiner and equalizer structure is considerably reduced as the number of diversity channels increases. In Figure 10, the average BER performance as a function of the normalized sampling rate, $\bar{f}$, for different values of the maximum Doppler frequency is illustrated for our ODCDFE(3,2) and Lo's receiver for the case of $S N R=20 \mathrm{~dB}$ and $L=1$. We see from the figure that our receiver structure is slightly better than Lo's model for high maximum Doppler frequencies and the normalized sampling rate less than $2,(\bar{f}<2)$. For low Doppler frequencies and $\bar{f} \geq 2$, the receiver structures perform almost identically. We also observe the similar effect as we increase the number of diversity channels. The main reason for this improvement is the use of the fractionally spaced matched filter for each diversity branch. Since the matched filter spans the whole CIR, we maximize the power of the current symbol.

\section{Conclusions}

A simplified antenna diversity combiner-equalizer structure was proposed and analyzed for CMR channel. This receiver is preferable to previously proposed approaches when CIR estimation rather than adaptive equalization is performed. Further research issues include "phase alignment" problem [11] which arises in CIR estimation for differentially encoded data and receiver design for systems with error control coding.

\section{REFERENCES}

[1] T. Eyceöz, "Reduced Complexity Optimal Diversity Combining and Adaptive Equalization Using Interpolated Channel Estimates with Applications to Cellular

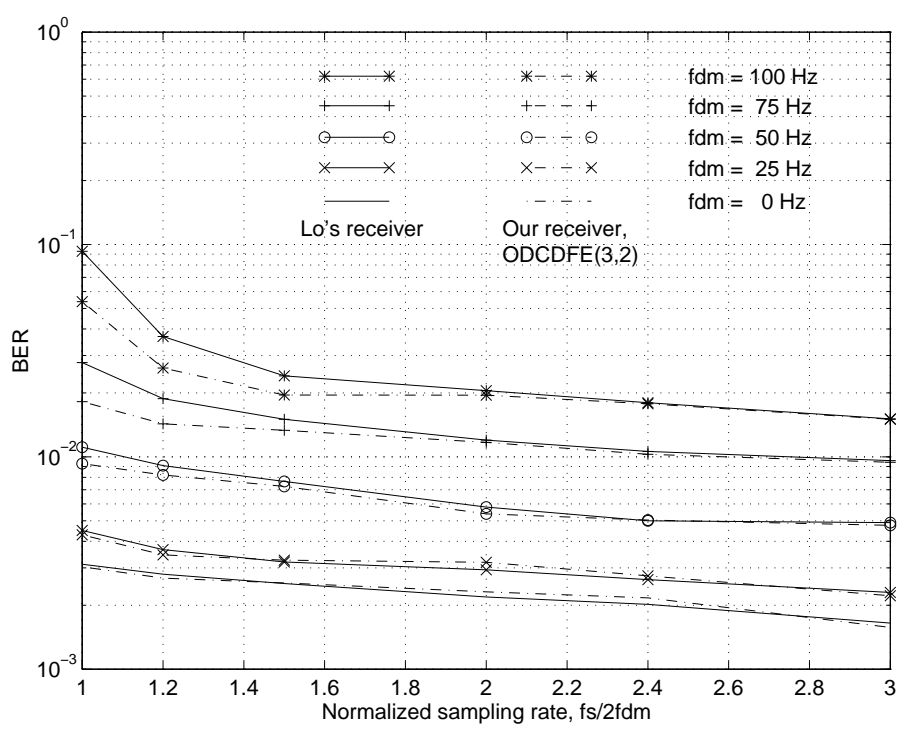

Figure 10: Performance comparison of our receiver structure and Lo's receiver structure as a function of the normalized sampling rate, $\bar{f}$, and the maximum Doppler frequency, $f_{d m}$ with $S N R=20 \mathrm{~dB}$ and $L=1$

Mobile Radio Channels", Master's thesis, North Carolina State Univ., November 1995.

[2] N. W. K. Lo, D. D. Falconer, and A. U. H. Sheikh, "Adaptive Equalization and Diversity Combining for a Mobile Radio Channel using Interpolated Channel Estimates", IEEE Transactions on Vehicular Technology, 40(3):636-645, August 1991.

[3] W. C. Jakes, Microwave Mobile Communications, John Wiley and Sons, New York, 1974.

[4] W. C. Y. Lee, Mobile Communications Engineering, McGraw-Hill, New York, 1982.

[5] J. G. Proakis, Digital Communications, McGraw-Hill, New York, 1995.

[6] S. Stein, "Fading Channel Issues in System Engineering", IEEE Journal on Selected Areas in Communications, 5(2):68-89, February 1987.

[7] P. Balaban and J. Salz, "Optimum Diversity Combining and Equalization in digital Data Transmission with Applications to Cellular Mobile Radio-Part I: Theoretical Considerations", IEEE Transactions on Communications, 40(5):885-894, May 1992.

[8] P. Dent, G. E. Bottomley, and T. Croft, "Jakes Fading Model Revisited", Electronics Letters, 29(13):11621163, June 1993.

[9] J. Salz, "Optimum Mean-Square Decision Feedback Equalization", Bell Systems Technical Journal, 52(8):1341-1373, October 1973.

[10] S. U. H. Qureshi, "Adaptive Equalization", Proceedings of the IEEE, 73(9):1349-1387, September 1985.

[11] R. D. Koilpillai, S. Chennakeshu, and R. L. Toy, "Low Complexity Equalizers for U.S. Digital Cellular System", Proceedings of VTC, 744-747, May 1992. 